Rapid Reviews COVID-19•

\title{
Review 2: "Infectivity and
}

immune escape of the new

SARS-CoV-2 variant of

interest Lambda"

\section{Orson Mestanza ${ }^{1}$}

${ }^{1}$ National Institute of Health Lab. Molecular Biology 734 LIMA, LIMA 51 PERU"

Published on: Aug 06, 2021

DOI: $10.1162 / 2 \mathrm{e} 3983 \mathrm{f5} .31803756$

License: Creative Commons Attribution 4.0 International License (CC-BY 4.0). 


\section{$\underline{\text { RR:C19 Evidence Scale rating by reviewer: }}$}

- Reliable. The main study claims are generally justified by its methods and data. The results and conclusions are likely to be similar to the hypothetical ideal study. There are some minor caveats or limitations, but they would/do not change the major claims of the study. The study provides sufficient strength of evidence on its own that its main claims should be considered actionable, with some room for future revision.

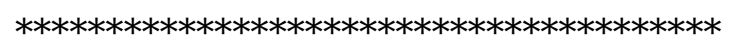

\section{Review:}

Methods: In the population of interest, it is not clear if they were previously infected by SARS-CoV-2 prior to receiving the vaccine. This should be clarified in the text. Further, the number of plasma samples collected is not specified. In the figure in the results section, there appear to be 79 plasma samples. However, this should also be noted in the text.

Results: Calculating the correlation analysis between convalescent plasma and neutralization may be important to consider. 